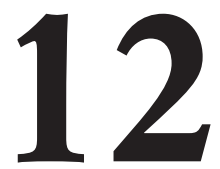

\title{
COMPETENCIAS GENÉRICAS EN LA UNIVERSIDAD. EVALUACIÓN DE UN PROGRAMA FORMATIVO
}

\section{(GENERIC SKILLS AT UNIVERSITY. EVALUATION OF A TRAINING PROGRAM)}

Paula Crespí

Universidad Francisco de Vitoria

José Manuel García-Ramos

Universidad Complutense de Madrid

DOI: $10.5944 / e d u c X X 1.26846$

Cómo referenciar este artículo/How to reference this article:

Crespí, P. y García-Ramos, J.M. (2021). Competencias genéricas en la universidad. Evaluación de un programa formativo. Educación XX1, 24(1), 297-327, http://doi.org/10.5944/ educXX1.26846

Crespí, P. \& García-Ramos, J.M. (2021). Generic skills at university. Evaluation of a training program. Educación XX1, 24(1), 297-327, http://doi.org/10.5944/educXX1.26846

\section{RESUMEN}

Las competencias genéricas han ido ganando relevancia en el entorno empresarial desde 1973, y posteriormente, desde el 2000, en el entorno de la educación superior. En este sentido, el EEES, manifiesta que la universidad ha de formar en competencias específicas y genéricas. La formación en competencias específicas ha sido bien asumida por las distintas asignaturas propias de cada grado. Sin embargo, no ocurre igual con las genéricas. Actualmente, en la universidad, estas competencias son desarrolladas indirectamente a través de asignaturas técnicas, de libre elección o de actividades formativas complementarias (AFC). Así, aunque el EEES reconoce la relevancia de la formación en estas competencias, la mayoría de las universidades no tienen asignaturas específicas para su desarrollo. 
Este estudio propone una asignatura obligatoria, específica y transversal a los distintos grados universitarios para desarrollar las competencias genéricas. Se pretende demostrar que la impartición de asignaturas específicas y regladas en materia de competencias genéricas es un método relevante para su desarrollo. Se diseñó un cuestionario sobre competencias genéricas satisfactoriamente válido y fiable. Este cuestionario se pasó a una muestra total de 610 estudiantes, de los cuales: 387 recibieron una formación específica en competencias genéricas y 223 no la recibieron. Los resultados obtenidos confirman las hipótesis planteadas acerca de la validez de la asignatura en la mejora significativa de las competencias genéricas, tanto en las intrapersonales, de superación personal y mirada profunda, como en las interpersonales, de comunicación y trabajo en equipo. Por tanto, se evidencia cómo las asignaturas sobre competencias genéricas se constituyen como un medio de enseñanza aprendizaje muy adecuado para su desarrollo. En este sentido, se sugiere una revisión de los planes de estudio si se quiere garantizar la adquisición y desarrollo de ambas competencias, específicas y genéricas, como aconsejan el EEES y el ámbito laboral.

\section{PALABRAS CLAVE}

Educación superior, competencias, competencias genéricas, asignatura, competencias intrapersonales, competencias interpersonales

\section{ABSTRACT}

Generic skills have gained increasing importance in the business world since 1973, and since the year 2000 in the area of higher education as well. Within this context the EHEA explicitly states that universities must educate both specific and generic skills. The education of specific skills has been well undertaken within the various courses of each degree program. However, the same cannot be said of generic skills. Currently at universities, these skills are developed indirectly through optional courses or complementary education activities (CEA). Thus, although the EHEA recognises the importance of learning these skills, the majority of universities do not offer specific courses for their acquisition.

This study proposes the creation of an obligatory and specific course, transversal to all university degrees, for the development of generic skills. The aim is to demonstrate that a structured and specific course on generic competences can be an important vehicle for their development. A valid and reliable questionnaire on generic skills was designed and administered 
to 610 students, of whom 387 received specific training in generic skills and 223 of whom did not. The results confirm the hypothesis regarding the effectiveness of a specific course in significantly enhancing the acquisition of generic skills, both intrapersonal, dealing with personal growth and introspection, and interpersonal, dealing with communication and teamwork. Evidence shows that specific courses on generic competences are an effective means of developing these skills. Thus, study programs should be revised to ensure student acquisition of both competences, specific and generic, as recommended by the EHEA and the employment market.

\section{KEY WORDS}

Higher education, skills, generic skills, course, intrapersonal skills, interpersonal skills

\section{INTRODUCCIÓN}

El EEES comienza a dar pasos en firme en 1999 a través de la Declaración de Bolonia. Esta pretende, por un lado, armonizar los distintos títulos universitarios de manera que estos puedan ser comparables, y por tanto, tengan la misma validez entre los distintos países de la Unión Europea; y por otro, pretende facilitar y asegurar una adecuada movilidad e intercambio entre los estudiantes de los países miembros (Declaración de Bolonia, 1999). Para avanzar en la creación del EEES y dar respuesta a la Declaración de Bolonia, surge el proyecto Tuning Educational Structures in Europe. González y Wagenaar (2006) proponen un diseño para implantar de manera armónica los distintos planes de estudio, respetando también sus diferencias. En esta armonización, se propone que los distintos planes de estudio trabajen sobre la línea de dos grandes marcos de referencia (González y Wagenaar, 2006): 1) Los resultados de aprendizaje, entendidos como lo que uno es capaz de manifestar que sabe o es capaz de hacer. 2) Las competencias, entendidas como el conjunto de conocimientos, destrezas, capacidades y valores.

Basándose en el proyecto Tuning (2006), Crespí (2019) recoge la tipología de competencias, integrando en ellas el enfoque educativo y laboral. Así, se puede hablar de dos tipos de competencias: las técnicas o específicas, aquellas propias de una asignatura, titulación, campo de conocimiento o profesión; y las transversales o genéricas, aquellas comunes a distintas asignaturas, titulaciones o profesiones, necesarias, en general, para la vida. Ejemplos de estas últimas competencias son el liderazgo, la iniciativa, la 
planificación, la resolución de problemas, la capacidad de comunicarse y de trabajar de manera cooperativa, entre otras.

En su propuesta, el proyecto Tuning afirma que las distintas titulaciones han de asegurar que los resultados de aprendizaje están orientados tanto a las competencias específicas como a las genéricas. Ambas han de ser la base de cualquier titulación universitaria. Además, añade que, la formación en competencias genéricas tiene un papel decisivo en la adecuada formación de los universitarios para su cometido en la sociedad, ya sea como profesionales o como ciudadanos (Almerich et al., 2018; Comisión Europea, 2017; González y Wagenaar, 2006; OECD, 2018; Pugh y Lozano, 2019; Sanjurjo, 2012; Villardón, 2015; UNESCO, 2015).

En el ámbito laboral, las competencias también cobran un papel decisivo en el desempeño de un puesto de trabajo, especialmente las de carácter transversal o genérico. Desde que McClelland (1973) propusiera testar competencias, en vez de la inteligencia y los conocimientos, como factor clave en el desempeño excelente en un puesto de trabajo o actividad concreta, las competencias se han ido introduciendo paulatinamente en la gestión empresarial; especialmente las denominadas soft skills. Observamos como las empresas quieren contratar, formar y promocionar a candidatos que tengan suficientemente desarrollas las competencias genéricas (soft skills). Así, consideran de menor importancia las técnicas (hard skills), pues estas son más fáciles de adquirir a través de la propia experiencia en el puesto de trabajo o a través de una formación específica (Alles, 2008, 2017; Boyatzis, 1982; Bunk, 1994; Carazo, 2012; García, 2018; González, 2017; Jericó, 2011; Olaz, 2018; Ramos, 2015, 2017; Spencer \& Spencer, 1993).

Por último, no podemos olvidar que la propia misión de la universidad, desde sus orígenes, aboga firmemente por una formación integral; es decir, por una sólida formación humana con una docencia centrada en la persona y en su proceso de aprendizaje; es decir, en enseñar a aprender. Una formación que pretende poner al alumno en camino de su propia excelencia personal y profesional. Esto implica desarrollar las competencias genéricas; además de las específicas (Baeten et al., 2013; Cardona et al., 2009; Domínguez, 2018; Esteban \& Román, 2016; Jaspers, 2013; Pérez, 2010).

Queda evidenciada pues, la importancia de que los alumnos universitarios reciban una educación que incluya la formación en competencias genéricas. ¿Pero, cómo desarrollan hoy los planes universitarios estas competencias? 
Actualmente, muchas universidades están haciendo un gran esfuerzo por introducir la formación en competencias en sus asignaturas y en sus planes de estudio; especialmente, este esfuerzo es notorio con respecto de las competencias específicas. En el caso de las competencias genéricas las universidades barajan, por lo general, tres modelos (Corominas et al., 2006; Crespí, 2020; Gijón, 2016; Villardón, 2015). Una opción consiste en que sean las propias asignaturas técnicas las que desarrollen las competencias genéricas. Esto implica que, profesores especializados en el desarrollo de las competencias técnicas, asumen también el desarrollo de las genéricas, pero de manera vehicular. Es decir, las competencias genéricas son desarrolladas como medio, y no como un fin en sí mismas. Otra opción pone de manifiesto que sean las asignaturas optativas las que desarrollen las competencias genéricas. En este caso, no se puede asegurar que todos los alumnos reciban esta formación. Una última opción contempla que sean las AFC las que desarrollen las competencias genéricas. En este caso, tampoco se puede afirmar que todos los alumnos reciban la formación.

En este sentido, se observa cierta incoherencia entre lo que propone el EEES y el ámbito laboral con relación a la realidad de los planes de estudio universitarios actuales. El proyecto Tuning, recoge la vital importancia del desarrollo y formación en competencias genéricas, críticas para los universitarios en sus futuras responsabilidades como ciudadanos y profesionales; sin embargo, en general, las universidades no cuentan con asignaturas obligatorias específicas para su desarrollo.

Esta investigación propone una cuarta opción, que consiste en el diseño de una asignatura obligatoria y transversal a los distintos planes de estudio, sobre competencias genéricas para los alumnos de primero de grado. De este modo, se lleva a cabo un estudio empírico que pretende demostrar la notable eficacia de esta asignatura curricular en el desarrollo de las competencias transversales, frente a las principales alternativas de desarrollo anteriormente descritas.

\section{OBJETIVO E HIPÓTESIS DE LA INVESTIGACIÓN}

El principal objetivo de este estudio es estudiar la eficacia de una asignatura específica sobre competencias genéricas como método para el desarrollo de dichas competencias. En este sentido, este estudio plantea contrastar las siguientes hipótesis de investigación $(\mathrm{H})$ :

- H1: Existen diferencias significativas en el nivel de competencias genéricas de los universitarios, en general, debido al efecto de la 
formación en dichas competencias, entre los que la reciben y los que no.

- H2: Existen diferencias significativas en el nivel de competencias genéricas de los estudiantes universitarios de Educación, debido al efecto de la formación, entre los que la reciben y los que no.

- H3: Existen diferencias significativas en el nivel de competencias genéricas de los estudiantes que reciben la formación dependiendo de la facultad en que estudian.

- H4: Existen diferencias significativas en el nivel de competencias genéricas de los estudiantes que reciben la formación dependiendo del sexo.

- H5: Existen diferencias significativas en el nivel de competencias genéricas de los estudiantes que reciben la formación debido a la interacción entre sexo y facultad.

\section{METODOLOGÍA}

\section{Diseño de la investigación y variables}

Esta investigación es de tipo cuasi-experimental. Recibir o no la formación constituye la variable independiente (VI) principal. Como VI secundarias: sexo, año de nacimiento, estudios y facultad. Como variables dependientes (VD), el grado de adquisición de las competencias genéricas: afán de superación, trabajo en equipo, mirada profunda y comunicación.

\section{Diseño de la asignatura}

La Universidad Francisco de Vitoria propone una asignatura para el desarrollo personal de sus alumnos a través de la adquisición de competencias genéricas. Una asignatura, impartida por expertos en la materia, obligatoria y común a las distintas titulaciones universitarias. Está diseñada ad hoc para desarrollar algunas de las competencias genéricas identificadas por Gonzalez y Wagenaar (2006). Competencias que contribuyen a que cada alumno, único e irrepetible, se ponga en camino de su propio proyecto de vida y de su plenitud personal y profesional (Crespí 2019).

El proyecto Tuning llega a establecer hasta 30 competencias genéricas críticas fruto de una encuesta entre empleadores, académicos y estudiantes. 
En cualquier caso, no se recomienda tratar de desarrollar a la vez, más que un número limitado de competencias (Benito y Cruz, 2006; Pugh y Lozano, 2019). Esta asignatura selecciona aquellas que considera más esenciales para empezar a desarrollar y consolidar en primero de grado (Tabla 1).

Tabla 1

Competencias genéricas objeto de la asignatura y su correspondencia de acuerdo al proyecto Tunning

\begin{tabular}{|c|c|c|}
\hline Tipología & $\begin{array}{l}\text { Competencias objeto de la } \\
\text { asignatura }\end{array}$ & $\begin{array}{l}\text { Correspondencia con la } \\
\text { clasificación enTuning }\end{array}$ \\
\hline \multirow{3}{*}{$\begin{array}{l}\text { Intraperso- } \\
\text { nales }\end{array}$} & $\begin{array}{l}\text { Mirada profunda, conocimiento } \\
\text { personal }\end{array}$ & $\begin{array}{l}\text { Genéricas - Interpersonales- in- } \\
\text { dividuales }\end{array}$ \\
\hline & Proactividad & $\begin{array}{l}\text { Genéricas - Sistémicas- capaci- } \\
\text { dad emprendedora }\end{array}$ \\
\hline & Resiliencia, superación personal & $\begin{array}{l}\text { Genéricas - Sistémicas- lide- } \\
\text { razgo }\end{array}$ \\
\hline \multirow{3}{*}{$\begin{array}{l}\text { Interperso- } \\
\text { nales }\end{array}$} & Comunicación interpersonal & $\begin{array}{l}\text { Genéricas - Instrumentales- lin- } \\
\text { güísticas }\end{array}$ \\
\hline & $\begin{array}{l}\text { Trabajo en equipo, resolución } \\
\text { de conflictos y negociación }\end{array}$ & Interpersonales- sociales \\
\hline & Liderazgo de servicio & Sistémicas- liderazgo \\
\hline \multirow{2}{*}{ Cognitivas } & $\begin{array}{l}\text { Toma de decisiones, planifica- } \\
\text { ción, gestión tiempo }\end{array}$ & $\begin{array}{l}\text { Genéricas - Instrumentales- me- } \\
\text { todológicas }\end{array}$ \\
\hline & Creatividad, innovación & $\begin{array}{l}\text { Genéricas - Sistémicas- capaci- } \\
\text { dad emprendedora }\end{array}$ \\
\hline
\end{tabular}

Ha de entenderse que no existen las competencias puras. De este modo, tampoco hay clasificaciones exactas sino distintas aproximaciones complementarias. Clasificar las competencias no tiene otro objetivo más que comprender mejor el tipo de competencia del que se está hablando.

La asignatura que se propone tiene como objetivo principal el desarrollo de las competencias de carácter intrapersonal, es decir, aquellas que se refieren principalmente a uno mismo, y las de carácter interpersonal, aquellas de relación, principalmente, con los demás. Por último, las competencias de carácter cognitivo, aquellas relacionadas principalmente con la capacidad de pensar, no son objeto de desarrollo directo. Es decir, se trabajan de manera vehicular como medio para desarrollar las principales.

Precisamente para poder desarrollar estos dos grandes tipos de competencias se articulan dos ámbitos bien diferenciados, que responden a la propia naturaleza de las competencias: la mentoría y la clase. 
La mentoría está dirigida a que el alumno adquiera competencias de carácter intrapersonal como la proactividad, la capacidad de asombro y el afán de superación. Consiste en un programa de desarrollo personal e individual en el que un mentor experto acompaña al alumno en este recorrido, a lo largo de 6 encuentros presenciales de una hora de duración.

La clase está dirigida a que el alumno desarrolle competencias de carácter interpersonal, como el trabajo en equipo y la comunicación. En este caso, son el docente y los demás compañeros quienes acompañan a cada estudiante en la adquisición de competencias, a través de clases temáticas-experienciales y del ABP (aprendizaje basado en proyectos) que han de llevar a cabo en equipo a lo largo de todo el curso.

La asignatura emplea metodologías activas y experienciales, centradas en el aprendizaje del alumno; convirtiéndole en el principal agente y protagonista de su desarrollo. Es precisamente el uso de estas metodologías el garante de que los alumnos adquieren y desarrollan de manera efectiva estas competencias, tan necesarias para su vida, personal y profesional.

Para una mejor comprensión de la asignatura, fundamentos, objetivos, temario, metodología, distribución de tiempos de trabajo y bibliografía de referencia, se facilita una guía docente de la asignatura (Anexo 1).

\section{Población y Muestra}

La población la configuran todos los alumnos universitarios de primer curso de carrera de la Comunidad de Madrid.

El tipo de muestra es incidental de dos universidades. Los universitarios de Educación de la U. Complutense de Madrid los tomamos como grupo control (GC), porque no reciben el tratamiento, la asignatura en competencias genéricas; y a los de la U. Francisco de Vitoria como grupo experimental (GE), ya que reciben la formación.

La muestra total la constituyen un total de 610 alumnos universitarios (en pretest), de los cuales, 223 alumnos no cursan la asignatura y 387 alumnos universitarios sí la cursan. Algo menor es la muestra en el postest, que queda constituida por 547 alumnos, de los que 193 no cursan la asignatura y 354 sí la cursan. Estos datos nos muestran, en ambos casos, un tamaño de muestra mayor al proporcionado por el tamaño muestral exigido, que es de 444, de los que 217 son para el GC y 227 para el GE. El tamaño de la muestra mínimo exigido fue calculado sobre una muestra finita, con una precisión 
del .40 y un nivel de confianza de .95 (programa ENE 2.2). A continuación, se muestra una tabla resumen de la muestra de la investigación (Tabla 2).

Tabla 2

Muestra de la investigación por facultades y universidades

\begin{tabular}{lccc}
\hline Facultad & $\begin{array}{c}\text { Muestra } \\
\text { mínima }\end{array}$ & $\begin{array}{c}\text { Muestra } \\
\text { pretest }\end{array}$ & $\begin{array}{c}\text { Muestra } \\
\text { postest }\end{array}$ \\
\hline Educación UCM & 217 & 223 & 193 \\
\hline Educación UFV & 18 & 58 & 60 \\
\hline Ciencias Jurídicas y Empresariales UFV & 50 & 66 & 60 \\
\hline Ciencias de la Salud UFV & 77 & 95 & 89 \\
\hline Experimentales UFV & 25 & 49 & 47 \\
\hline Escuela Politécnica Superior UFV & 12 & 47 & 45 \\
\hline Ciencias de la Comunicación UFV & 45 & 72 & 53 \\
\hline Total & $\mathbf{4 4 4}$ & $\mathbf{6 1 0}$ & $\mathbf{5 4 7}$ \\
\hline
\end{tabular}

\section{Instrumento}

Para medir el desarrollo de las principales competencias objeto de la asignatura, se diseñó un instrumento específico. Para ello, se consultaron diversas fuentes específicas en materia de evaluación de competencias genéricas (Álvarez et al., 2011; Morales et al., 2013; Morillas, 2012; Muñoz et al., 2011; Pérez et al., 2010; Pozo, 2017; Ruiz et al., 2017).

El cuestionario resultante evalúa las principales dimensiones y subdimensiones de las competencias genéricas objeto de la asignatura descritas (Tabla 3):

Tabla 3

Dimensiones, subdimensiones e indicadores del cuestionario sobre competencias genéricas

\begin{tabular}{lllc}
\hline Dimensión & Subdimensión & Indicador & Ítem \\
\hline & $\begin{array}{l}\text { Mirada } \\
\text { profunda }\end{array}$ & $\begin{array}{l}\text { Conocimiento, aceptación y gestión } \\
\text { personal }\end{array}$ & 9 \\
Intrapersonal & $\begin{array}{l}\text { Superación } \\
\text { Personal }\end{array}$ & $\begin{array}{l}\text { Búsqueda de sentido, orientación a } \\
\text { la excelencia y proactividad }\end{array}$ & 9 \\
\hline
\end{tabular}




\begin{tabular}{lllc}
\hline Dimensión & Subdimensión & Indicador & Ítem \\
\hline & $\begin{array}{l}\text { Trabajo en } \\
\text { Equipo }\end{array}$ & $\begin{array}{l}\text { Trabajo cooperativo, gestión del } \\
\text { clima, orientación a resultados }\end{array}$ & 9 \\
Interpersonal & Comunicación & $\begin{array}{l}\text { Comunicación verbal, para verbal y } \\
\text { no verbal. Comunicación para el en- } \\
\text { cuentro (empatía, asertividad y es- } \\
\text { cucha) }\end{array}$ & 9 \\
\hline
\end{tabular}

Como se puede observar, la dimensión intrapersonal alude a dos grandes competencias: la mirada profunda, relacionada a su vez con las competencias de conocimiento, aceptación y gestión personal; y la superación personal, relacionada con la búsqueda de sentido, la orientación a la excelencia y la proactividad. Por otro lado, la dimensión interpersonal alude a las competencias de trabajo en equipo, relacionada a su vez con las competencias de trabajo cooperativo, gestión del clima y orientación a resultados; y de comunicación, que recoge la comunicación verbal, no verbal y otras competencias críticas para el encuentro como la empatía, la asertividad y la escucha.

Este cuestionario no refleja las competencias cognitivas de manera directa porque no son objeto principal de la asignatura; aunque sí lo hace de manera indirecta en alguno de los ítems, ya que como hemos dicho estas competencias son vehiculares de las principales.

De este modo, el cuestionario plantea 41 ítems, de los cuales, los 4 primeros recogen datos asociados a la identificación del estudiante, los 36 siguientes se corresponden con las dimensiones, 9 para cada subdimensión, y el último ítem es un ítem criterio. Este cuestionario recoge respuestas tipo Likert, del 1 al 6, donde el 1 indica nunca o nada y el 6, siempre o completamente.

Una vez diseñado nuestro cuestionario, 18 expertos en investigación educativa y en competencias procedieron a validarlo. Los expertos se seleccionaron por su prestigio en la materia en el ámbito académico, tanto privado como público; algunos de ellos también contaban con experiencia laboral en el ámbito de la psicología y de los recursos humanos. El cuestionario para la valoración de expertos solicitaba un análisis exhaustivo de todos los ítems en torno a los criterios de coherencia con la dimensión, sencillez en la redacción y ajuste a los destinatarios. También solicitaba un análisis en términos generales del cuestionario para valorar el contenido, el número de ítems y el orden lógico. Los resultados muestran unas valoraciones medias de todos los ítems, superiores a 5.17, tanto en las dimensiones como en las subdimensiones. Además, los distintos valores que 
toma el coeficiente de variación de Pearson, siempre cercano a cero, indican homogeneidad en las respuestas de los expertos.

En cualquier caso, tanto del análisis cualitativo como del cuantitativo se tomaron observaciones para realizar pequeñas mejoras que se incorporaron a la elaboración del cuestionario final (Anexo 2).

\section{Procedimiento de recogida y análisis de datos}

El cuestionario se pasó a la muestra identificada siguiendo un mismo protocolo de actuación e indicaciones para todos los estudiantes y grupos (GE y GC). El pretest se pasó al inicio del curso 16-17 y el postest al final de este. En el análisis estadístico se utilizó el programa IBM SPSS versión 20. Para la validación del cuestionario y de sus dimensiones se calcularon Coeficientes de Fiabilidad Alfa de Cronbach; para la validez criterial convergente, el coeficiente de correlación de Pearson; y para el estudio de ítems, se calcularon índices de homogeneidad (IH) e índices de validez (IV). Se realizaron análisis factoriales exploratorios en los que se emplearon distintos procedimientos de factorización como ACP (Análisis de Componentes Principales), MV (Máxima Verosimilitud) y de rotación (Promax y Oblimin). Por último, para los análisis diferenciales y contraste de las hipótesis se emplearon: $t$ de Student, F de Anova, pruebas post hoc (Scheffé), así como estimaciones del Tamaño del Efecto en ambos casos.

\section{RESULTADOS}

\section{Validación del cuestionario}

Alfa de Cronbach nos indica una buena valoración de la fiabilidad del instrumento de medida y de sus dimensiones. El análisis de validez criterial convergente, a través de los coeficientes de correlación de Pearson, mostró significativas y relevantes correlaciones lineales (Tabla 4).

Tabla 4

Análisis de fiabilidad y validez criterial del instrumento (postest)

\begin{tabular}{lcccccc}
\hline & DIM & DIM & SUBDIM & SUBDIM & SUBDIM & SUBDIM \\
& $\mathbf{1}$ & $\mathbf{2}$ & $\mathbf{1}$ & $\mathbf{2}$ & $\mathbf{3}$ & $\mathbf{4}$ \\
\hline Fiabilidad (rxx) & .90 & .90 & .83 & .83 & .83 & .86 \\
Validez (rxy) & .64 & .65 & .60 & .59 & .54 & .63 \\
$\mathrm{~N}^{\circ}$ ítems & 18 & 18 & 9 & 9 & 9 & 9 \\
\hline
\end{tabular}


Los análisis de homogeneidad y validez de los ítems muestran valores satisfactorios para todos ellos, valores mayores a .20 en todos los casos. Para estudiar la relación entre la estructura empírica y la teórica del cuestionario, se realizaron una serie de análisis factoriales exploratorios; el último realizado, en el que "forzamos" la solución a cuatro factores, muestra unos resultados que avalan la existencia de una clara relación entre la estructura empírica de cuatro factores y la teórica de cuatro subdimensiones.

\section{Análisis descriptivos y correlacionales}

Se realizaron análisis descriptivos para observar el comportamiento de las variables en las muestras para las cuatro subdimensiones, las dos dimensiones y la puntuación total, en pretest y en postest, tanto para las distintas universidades, sus facultades y la variable sexo. Estos análisis muestran que las medias del postest parecen ser superiores a las del pretest en todos los casos para las distintas facultades y sexos.

\section{Análisis diferenciales}

Teniendo en cuenta que el objetivo principal de esta investigación consiste en demostrar la eficacia formativa de una asignatura específica sobre competencias genéricas, se presentan, a continuación, los análisis realizados para el contraste de las hipótesis.

La primera hipótesis afirma la existencia de diferencias en el nivel competencial debido al efecto de la formación entre los estudiantes que recibieron la formación y los que no (Tabla 5).

Tabla 5

Diferencias significativas a favor de los alumnos que cursaron el programa

\begin{tabular}{lcccccc}
\hline $\begin{array}{l}\text { Competencias } \\
\text { (VD) }\end{array}$ & $\begin{array}{c}\text { Media } \\
\text { Postest } \\
\text { GE }\end{array}$ & $\begin{array}{c}\text { Media } \\
\text { Postest } \\
\text { GC }\end{array}$ & $\begin{array}{c}\text { Difer. me- } \\
\text { dias Postest } \\
\text { GE-GC }\end{array}$ & $\begin{array}{c}\boldsymbol{t} \text { de } \\
\text { Student }\end{array}$ & $\begin{array}{c}\text { Sig } \\
\text { (bilate- } \\
\text { ral) }\end{array}$ & $\begin{array}{c}\text { TE. eta } \\
\text { cuadrado } \\
\text { parcial }\end{array}$ \\
\hline Total & 181.98 & 161.17 & 20.81 & 13.64 & .000 & .25 \\
D. Intrapersonal & 91.32 & 78.59 & 12.73 & 15.78 & .000 & .31 \\
D. Interpersonal & 90.66 & 82.58 & 8.08 & 9.56 & .000 & .14 \\
S. Mirada & 45.64 & 39.72 & 5.92 & 13.18 & .000 & .24 \\
S. Superación & 45.68 & 38.87 & 6.81 & 15.13 & .000 & .30 \\
S. T. Equipo & 45.74 & 42.89 & 2.85 & 5.97 & .000 & .07 \\
S. Comunicación & 44.92 & 39.69 & 5.23 & 10.56 & .000 & .17 \\
\hline
\end{tabular}


Los análisis indican que todos los alumnos del GE mejoran su nivel de desarrollo para cada competencia (VD). La media del postest de los alumnos que cursaron la asignatura es mucho mayor a la de los que no la cursaron. La diferencia varía entre un máximo de 20.81 para la puntuación total y un mínimo de 2.85 para la VD "trabajo en equipo". Se comprobó que en el pretest no había diferencias significativas entre ambos grupos (GC y GE).

En el postest, los resultados de la $t$ de Student y el nivel de significación nos indican diferencias significativas debido al efecto formación en el nivel de progreso de todas las competencias (VD), a favor de los alumnos que reciben la formación. También observamos que, en todos los casos, el tamaño del efecto (TE) es relevante. Valores comprendidos entre .14 y .31. De este modo, podemos afirmar que los alumnos que reciben la formación en competencias genéricas aumentan significativamente su desarrollo en las competencias de "mirada profunda", "superación personal", "trabajo en equipo" y "comunicación”, frente a los que no la reciben.

La segunda hipótesis afirma que encontraremos diferencias significativas en el nivel competencial debido al efecto de la formación entre los estudiantes de Educación que recibieron la formación y los que no (Tabla 6).

Tabla 6

Diferencias significativas a favor de los estudiantes de Educación que cursaron la formación

\begin{tabular}{lcccccc}
\hline $\begin{array}{l}\text { Competencias } \\
\text { (VD) }\end{array}$ & $\begin{array}{c}\text { Media } \\
\text { Postest } \\
\text { GE }\end{array}$ & $\begin{array}{c}\text { Media } \\
\text { Postest } \\
\text { GC }\end{array}$ & $\begin{array}{c}\text { Difer. me- } \\
\text { dias Postest } \\
\text { GE-GC }\end{array}$ & $\begin{array}{c}\boldsymbol{t} \text { de } \\
\text { Student }\end{array}$ & $\begin{array}{c}\text { Sig } \\
\text { (bilate- } \\
\text { ral) }\end{array}$ & $\begin{array}{c}\text { TE. eta } \\
\text { cuadrado } \\
\text { parcial }\end{array}$ \\
\hline Total & 188.82 & 161.17 & 27.65 & 11.19 & .000 & .33 \\
D. Intrapersonal & 94.83 & 78.59 & 16.24 & 12.67 & .000 & .39 \\
D. Interpersonal & 93.98 & 82.58 & 11.40 & 9.08 & .000 & .20 \\
S. Mirada & 47.55 & 39.72 & 7.83 & 10.37 & .000 & .30 \\
S. Superación & 47.28 & 38.87 & 8.41 & 11.58 & .000 & .35 \\
S. T. Equipo & 47.93 & 42.89 & 5.05 & 7.74 & .000 & .14 \\
S. Comunicación & 46.05 & 39.69 & 6.36 & 8.52 & .000 & .18 \\
\hline
\end{tabular}

Los análisis nos indican que todos los alumnos del GE mejoran su nivel de desarrollo para cada competencia (VD). La media del postest de los alumnos que cursaron la asignatura es bastante mayor a la de los que no la cursaron. La diferencia varía entre un máximo de 27.65 para la puntuación total y un mínimo de 5.05 para el "trabajo en equipo". Se comprobó que en el pretest no había diferencias significativas entre ambos grupos (GC y GE). 
En el postest, las $t$ de Student muestran que existen diferencias significativas debido al efecto formación en el nivel de progreso de todas las competencias (VD), a favor de los estudiantes de Educación que reciben la formación. Además, observamos que el TE, para todos los supuestos, es relevante; y varía en valores comprendidos entre .14 y .39. De este modo, podemos afirmar que los estudiantes de Educación que han cursado la formación han aumentado significativamente el nivel de adquisición de todas las competencias genéricas estudiadas con respecto de los alumnos que no la han cursado.

La tercera hipótesis afirma que habrá diferencias significativas entre los estudiantes que cursan la asignatura debido a la facultad en que cursan sus estudios (Tabla 7).

Tabla 7

Diferencias significativas entre los alumnos que cursaron el programa en función del tipo de estudios

\begin{tabular}{lccc}
\hline $\begin{array}{l}\text { Competencias } \\
\text { (VD) }\end{array}$ & $\begin{array}{c}\text { F } \\
\text { ANOVA }\end{array}$ & $\begin{array}{c}\text { Sig. } \\
\text { (bilateral) }\end{array}$ & $\begin{array}{c}\text { TE. } \\
\text { eta cuadrado parcial }\end{array}$ \\
\hline Total & 4.27 & .000 & .06 \\
D. Intrapersonal & 4.95 & .000 & .07 \\
D. Interpersonal & 2.91 & .010 & .04 \\
S. Mirada & 4.31 & .000 & .06 \\
S. Superación & 4.30 & .000 & .06 \\
S. T. Equipo & 3.53 & .000 & .05 \\
S. Comunicación & 1.98 & .080 & - \\
\hline
\end{tabular}

Los análisis nos indican que hay diferencias de medias en todas las VD entre las distintas facultades (Educación, Salud, Jurídicas y Empresariales, Experimentales, Politécnica y Comunicación). Se comprobó, a través de varios ANOVAS, que en el pretest no había diferencias significativas entre las distintas facultades.

En el postest, la F de Anova y su nivel de significación nos muestran la existencia de diferencias significativas entre los universitarios que recibieron la formación dependiendo del grado. Estas diferencias, se dan en todas las competencias (VD) menos en "comunicación". Para determinar entre qué facultades se hallan las diferencias realizamos la prueba post hoc (Scheffé). Comprobamos que las diferencias significativas entre facultades se dan en la facultad de Ciencias de la Salud y en la Escuela Politécnica, con respecto de la de Educación; siempre a favor de esta última. Por último, el TE se sitúa en valores medios, moderados y débiles; ya que varía entre .07 y .04. 
Si bien es cierto que existen diferencias menores respecto de la dimensión interpersonal (TE .04) y la subdimensión de "trabajo en equipo" (TE .05).

La cuarta hipótesis afirma que habrá diferencias significativas entre los estudiantes que cursan la asignatura en función al sexo (Tabla 8).

Tabla 8

Diferencias significativas entre los alumnos que cursaron la asignatura dependiendo del sexo

\begin{tabular}{lcccccc}
\hline $\begin{array}{l}\text { Competencias } \\
\text { (VD) }\end{array}$ & $\begin{array}{c}\text { Media } \\
\text { Postest } \\
\text { Mujeres } \\
(\mathbf{M})\end{array}$ & $\begin{array}{c}\text { Media } \\
\text { Postest } \\
\text { Hombres } \\
(\mathbf{H})\end{array}$ & $\begin{array}{c}\text { Diferencia } \\
\text { Media } \\
\text { Postest } \\
\text { M-H }\end{array}$ & $\begin{array}{c}\text { F } \\
\text { ANOVA }\end{array}$ & $\begin{array}{c}\text { Sig. } \\
\text { (bilate- } \\
\text { ral) }\end{array}$ & $\begin{array}{c}\text { TE. eta } \\
\text { cuadrado } \\
\text { parcial }\end{array}$ \\
\hline Total & 184.67 & 175.85 & 8.82 & 21.53 & .000 & .06 \\
D. Intrapersonal & 92.54 & 88.56 & 3.97 & 14.68 & .000 & .04 \\
D. Interpersonal & 92.14 & 87.29 & 4.85 & 23.55 & .000 & .06 \\
S. Mirada & 46.02 & 44.79 & 1.23 & 4.73 & .031 & .01 \\
S. Superación & 46.52 & 43.78 & 2.74 & 24.10 & .000 & .06 \\
S. T. Equipo & 46.62 & 43.74 & 2.88 & 29.73 & .000 & .08 \\
S. Comunicación & 45.52 & 43.55 & 1.97 & 10.88 & .000 & .03 \\
\hline
\end{tabular}

Los análisis nos indican que hay diferencias de medias en el postest entre mujeres y hombres, a favor, en todos los casos, de la mujer, y para todas las VD (competencias). Se comprobó, a través de varios ANOVAS, que en el pretest no había diferencias significativas entre ambos sexos, ni en el GE, ni en el GC. Y tampoco había diferencias significativas entre pretest y postest en el GC.

En el postest, la F de Anova y su nivel de significación muestran que para todas las VD hay diferencias significativas entre los estudiantes que recibieron la formación dependiendo de si es hombre o mujer. El TE se sitúa en valores medios, moderados y débiles; y varía en valores comprendidos entre .08 y .01. De este modo, podemos afirmar que existen diferencias entre los alumnos que han cursado la asignatura, a favor de la mujer. En concreto, existen diferencias en las competencias de "mirada profunda", "superación personal", "trabajo en equipo" y "comunicación"; en el caso de la dimensión interpersonal (TE .04), la subdimensión de "mirada profunda" (TE .01) y la subdimensión de "comunicación" (TE .03), las diferencias son menores.

La quinta hipótesis afirma que encontraremos diferencias significativas entre los estudiantes que cursan la asignatura por efecto de la interacción entre el sexo y la facultad (Tabla 9). 
Tabla 9

Diferencias entre los alumnos que cursaron la formación por efecto de la interacción entre sexo y facultad

\begin{tabular}{lcc}
\hline $\begin{array}{l}\text { Competencias } \\
\text { (VD) }\end{array}$ & $\begin{array}{c}\text { F } \\
\text { ANOVA }\end{array}$ & $\begin{array}{c}\text { Sig. } \\
\text { (bilateral) }\end{array}$ \\
\hline Total & 1.79 & .110 \\
D. Intrapersonal & 1.74 & .120 \\
D. Interpersonal & 1.82 & .110 \\
S. Mirada & 1.67 & .140 \\
S. Superación & 1.33 & .250 \\
S. T. Equipo & 1.11 & .350 \\
S. Comunicación & 1.96 & .080 \\
\hline
\end{tabular}

En este caso, la F de Anova y su nivel de significación no nos muestran diferencias significativas entre los estudiantes que cursan la asignatura por efecto de la interacción entre el sexo y la facultad. El nivel de competencias no varía en ninguna VD diferencialmente entre sexos, en presencia de una facultad u otra. Como vimos anteriormente, las mujeres desarrollaron mejor todas las competencias, y ahora vemos que, independientemente de la facultad en que cursaron sus estudios.

\section{DISCUSIÓN}

El EEES, a través del proyecto Tuning, propone que los planes de estudio trabajen en torno a resultados de aprendizaje y competencias, tanto específicas como genéricas; competencias, estas últimas, indispensables para la vida en general (Almerich et al., 2018; Alonzo et al., 2016; González y Wagenaar, 2006; OECD, 2018; Pugh y Lozano, 2019). Por otro lado, en el ámbito laboral se recoge el impacto que tienen las soft skills (competencias genéricas) en el desempeño excelente de un trabajador respecto de sus funciones o tareas, así como en la selección y progreso de su carrera profesional (Alles, 2017; García, 2018; González, 2017; Olaz, 2018). En este contexto educativo y laboral, apreciamos un esfuerzo por parte de algunas universidades a la hora de incorporar de alguna manera el desarrollo de las competencias genéricas, si bien, como veíamos, el esfuerzo es más notorio con respecto de las específicas. Parece que el tratamiento vehicular que tienen las competencias genéricas en las asignaturas técnicas, optativas o de AFC, aunque pueda tener cierto impacto en la mejora competencial de los estudiantes (Gijón, 2016; Jauregui, 2018; Villardón, 2015), es significativamente menor al obtenido a través de las asignaturas específicas en competencias genéricas (H1 y H2) (Crespí, 2019, 2020). Los universitarios 
que han recibido la formación en competencias genéricas desarrollan un nivel competencial significativamente superior en "trabajo en equipo", "mirada", "afán de superación" y "comunicación", a los que no la han recibido. También quisimos contrastar si entre facultades se daban o no diferencias (H3). En este sentido, encontramos que, de todas las facultades que cursaron la asignatura, los estudiantes de la facultad de Educación obtuvieron un mayor nivel de desempeño en todas las competencias medidas, menos en el caso de "comunicación" con respecto de la facultad de Ciencias de la Salud y de la Politécnica. Parece que tenga sentido que los alumnos de Educación, por el propio interés intrínseco y vocacional en la formación de sus futuros alumnos, puedan entender y aprovechar mejor una asignatura que les capacita como futuros docentes. También observamos que hay un distinto nivel de aprendizaje en función del sexo (H4); las mujeres parecen ser más sensibles a este tipo de formación. Esto puede deberse a que algunas de estas competencias están asociadas a funciones del hemisferio derecho del cerebro, para el cual las mujeres tienen una mayor predisposición innata. En cualquier caso, estas dos últimas hipótesis (H3 y H4) recogen diferencias menores, aunque significativas, debido a que el TE es débil y moderado, algo que se reafirma al no encontrar diferencias significativas en la interacción entre sexo y facultad (H5).

En esta formación específica es clave contar con profesionales expertos en el desarrollo de competencias genéricas, así como trabajar sobre la base de una metodología experiencial (Gómez, 2018; Kolb, 2015). Y es que, si bien es cierto que la experiencia cobra un lugar esencial en cualquier aprendizaje, más aún en el aprendizaje de competencias genéricas, del que se espera que este se transfiera a la realidad, torne en acciones o comportamientos y estos en hábitos buenos y estables. Otra clave para este tipo de asignaturas es hacer hincapié en el saber (conocimientos) y en el saber hacer (habilidades) con el enfoque integrador del saber ser (valores y actitud). Así, aunque este estudio ha partido inicialmente de la definición que hace Tuning de competencias, esta asignatura trabaja sobre la base de otra definición con carácter más integrador entre el enfoque académico y el laboral como solicitan algunos autores (Gijbels, 2011; Martínez et al., 2019). Así, trabajamos las competencias personales entendidas como "el conjunto dinámico de conocimientos (saber), habilidades o destrezas (saber hacer), actitudes y valores (saber ser) que, interiorizados y encarnados en nuestros actos, comportamientos o maneras de hacer, nos ponen en el camino de nuestra propia madurez, excelencia, plenitud y felicidad" (Crespí, 2019, p. 98).

Por último, pese al valor que se da a las competencias genéricas en la educación y en lo laboral, la universidad, en términos generales, las desarrolla de manera colateral y secundaria. No suele contar con asignaturas específicas y curriculares impartidas por expertos en competencias genéricas 
que trabajan con metodologías activas. Esto pone en evidencia una grave incoherencia entre la teoría (marco del EEES y el laboral) y la práctica (la realidad de los planes de estudio). Se manifiesta, por tanto, la relevancia de ofrecer una formación de calidad que proporcione a los estudiantes las competencias genéricas necesarias para su desarrollo (Martínez y González, 2019, Martínez et al., 2019; Pugh y Lozano, 2019).

\section{CONCLUSIONES}

La enseñanza en materia de competencias genéricas en la universidad tiene aún un largo camino por recorrer, para que la educación que reciben los estudiantes sea una educación integral y adecuada, de modo que cada uno de ellos, respondiendo a su vocación, pueda también dar respuesta a lo que el mundo espera de ellos. Este estudio ha abordado sustancialmente la importancia de forjar competencias genéricas, competencias que son requeridas tanto a nivel académico como a nivel profesional. Este trabajo de investigación aporta varios aspectos relevantes en el desarrollo y formación de las competencias genéricas. En primer lugar, se ofrece el diseño de una formación específica para mejorar el nivel de desempeño de las competencias genéricas, intra e interpersonales. Se aporta también una propuesta de definición más integradora del concepto de competencias que recoge tanto el enfoque educativo como el profesional. En segundo lugar, se ofrece un estudio empírico que demuestra que los estudiantes que han recibido la formación específica en competencias genéricas, las desarrollan significativamente, en mejor nivel que los que no la recibieron. Esto permite concluir con varias afirmaciones. Por un lado, podemos decir que se pueden diseñar programas universitarios curriculares, eficaces y válidos que permitan a los estudiantes desarrollar las competencias transversales. En concreto, hemos podido validar un programa de formación, como asignatura que permite formar y adquirir las competencias genéricas, intra e interpersonales. Por otro lado, los alumnos que han cursado la formación específica mejoran en todas las competencias genéricas objeto de estudio de manera significativa con respecto de los que no la han cursado ( $\mathrm{H} 1 \mathrm{y}$ H2). Respecto de los que han cursado la asignatura, parece que el grupo de los estudiantes de educación (H3) y el grupo de las mujeres (H4) son más sensibles a este tipo de formación.

Consideramos esta propuesta novedosa, ya que, a través de una asignatura diseñada ad hoc para ello, se han conseguido resultados significativos y con altos tamaños de efecto; y es relevante, ya que los resultados de este estudio pueden aportar luz en el marco teórico de cómo desarrollar este tipo de competencias. Se recoge aquí también una clara apuesta por ir generando una formación universitaria más completa y de 
mayor calidad, que facilite a los alumnos un mejor desarrollo personal, académico y profesional. Quizás este trabajo de investigación pueda ser un punto de partida para que la comunidad universitaria empiece a reflexionar acerca de la relevancia de incluir este tipo de formación en los programas de estudio universitarios. El objetivo es formar y desarrollar las competencias transversales, que contribuyen a la formación integral, siguiendo el planteamiento indicado por el EEES y saliendo al encuentro de la inquietud empresarial acerca de la importancia de forjar las soft skills. Ojalá estas recomendaciones puedan servir a la universidad para que valore la importancia de formar este tipo de competencias que, yendo más allá de los conocimientos y las técnicas, pretenden formar integralmente a sus alumnos, preparándolos mejor para dar respuesta a su vida, en el ejercicio de su vocación. 


\section{REFERENCIAS BIBLIOGRÁFICAS}

Alles, M.A. (2008). Desempeño por competencias: evaluación de $360^{\circ}\left(2^{\mathrm{a}}\right.$ ed.). Granica.

Alles, M.A. (2017). Elija al mejor. La entrevista en selección de personas. La entrevista por competencias. Granica.

Almerich, G., Díaz, I., Cebrián, S., y Suárez, J. (2018). Estructura dimensional de las competencias del siglo XXI en alumnado universitario de educación. RELIEVE, 24(1), art. 5. https://doi.org/10.7203/ relieve.24.1.12548

Alonzo, D.L., Valencia, M.C., Vargas, J.A., Bolívar, N.J., y García, M.J. (2016). Los estilos de aprendizaje en la formación integral de los estudiantes. Revista Boletín Redipe, 5(4), 109-114.

Álvarez, M., Bisquerra, R., Filella, G., Fita, E., Martínez, F., y Pérez, N. (2011). Diseño y evaluación de programas de educación emocional. Wolters Kluwer.

Baeten, M., Struyven, K., \& Dochy, F. (2013). Student-centered teaching methods: Can they optimize students' approaches to learning in professional higher education? Studies in Educational Evaluation, 39(1), 14-22. https://doi.org/10.1016/j. stueduc.2012.11.001

Benito, A. y Cruz, A. (2006). Nuevas claves para la Docencia Universitaria en el Espacio europeo de Educación Superior. Narcea.

Boyatzis, R. (1982). The Competent Manager: A Model for Effective Performance. John Wiley y Sons.

Bunk, G.P. (1994). La transmisión de competencias en la formación y perfeccionamiento profesionales de la RFA. Revista Europea de Formación Profesional, 1, 8-14.

Carazo, J.A. (2012). Universidad Ferrovial SUMMA: una fábrica de talento y liderazgo. Capital humano: revista para la integración y desarrollo de los recursos humanos, 25(267), 5257.

Cardona, A., Barrenetxea, M., Mijangos, J., y Olaskoaga, J. (2009). Concepto y determinantes de la calidad en la educación superior. Un sondeo de opinión entre profesores de universidades españolas. Archivos Analiticos de Políticas Educativas, 17(10), 1-25.

Comisión Europea (2017). Comunicación de la Comisión al Parlamento Europeo, al Consejo, al Comité Económico y Social Europeo y al Comité de las Regiones sobre una agenda renovada de la UE para la educación superior. https://bit.ly/2OzU4gU

Corominas, E., Tesouro, M., Capell, D., Teixidó, J., Pèlach, J., y Cortada, R. (2006). Percepciones del profesorado ante la incorporación de las competencias genéricas en formación universitaria. Revista de educación, 341, 301-336.

Crespí, P. (2019). La necesidad de una formación en competencias personales transversales en la universidad. Diseño y evaluación de un programa de formación. Fundación Universitaria Española.

Crespí, P. (2020). How higher education can develop generic competences? IJAEDU International e-Journal of Advances in Education, VI(16). https:// doi.org/10.18768/ijaedu.616003 
Declaración de Bolonia. (1999). Bolonia. https://bit.ly/3eHcXZZ

Domínguez, X.M. (2018). Ética del docente. Fundación Emmanuel Mounier.

Esteban, F. y Román, B. (2016). ¿Quo vadis, universidad? Editorial Universitat Oberta de Catalunya.

García, N.C. (2018). Evaluación del Desempeño del Talento Humano Basado en Competencias: Evaluación por Competencias, Desarrollo del Capital Humano. Editorial Académica Española.

Gijbels, D. (2011). Assessment of vocational competence in higher education: reflections and prospects. Assessment and Evaluation in Higher Education, 36(4), 381-383. https://doi. org/10.1080/02602938.2011.581859

Gijón, J. (Coord.), (2016). Formación por competencias y competencias para la formación. Perspectivas desde la investigación. Síntesis.

González, A.L. (2017). Métodos de compensación basados en competencias (3 ed.). Editorial Universidad del Norte.

González, J. y Wagenaar, R. (2006). Tuning Educational Structures in Europe II. La contribución de las universidades al Proceso de Bolonia. Universidad de Deusto.

Gómez, J. (2018). El aprendizaje experiencial. Capacitación y desarrollo en las organizaciones. Universidad de Buenos Aires.

Jaspers, K. (2013). La idea de la universidad. Ediciones Universidad de Navarra.

Jauregui, T. (2018). Estrategia Didáctica para Fortalecer las Competencias
Genéricas En Educación Superior. Editorial Académica Española.

Jericó, P. (2011). La nueva gestión del talento: construyendo compromiso. Pearson Educación.

Kolb, D.A. (2015). Experiential Learning: Experience as the Source of Learning and Development. Pearson Education.

Martínez, P. y González, C., (2019). Competencias personales y participativas vinculantes a la inserción laboral de los universitarios: Validación de una escala. RELIEVE, 25(1), art. 6. https://doi.org/10.7203/ relieve.25.1.13164

Martínez, P., González, C., y Rebollo, N. (2019). Competencias para la empleabilidad: un modelo de ecuaciones estructurales en la Facultad de Educación. Revista de Investigación Educativa, 31(1), 57-73. https://doi.org/10.6018/rie.37.1.343891

McClelland, D.C. (1973). Testing for Competence Rather Than for "Intelligence". American Psychologist, 28(1), 1-14.

Morales, M., Benítez, M., y Agustín, D. (2013). Habilidades para la vida (cognitivas y sociales) en adolescentes de una zona rural. Revista Electrónica de Investigación Educativa, 15(3), 98113.

Morillas, M.D. (Coord.) (2012). Competencias para la ciudadania: reflexión, decisión, acción. Narcea.

Muñoz, C., Crespí, P., y Angrehs, R. (2011). Habilidades sociales. Ediciones Paraninfo.

OECD. (2018). Education 2030: The Future of Education and Skills. Position paper. https://bit.ly/2CpBOV2 
Olaz, A.J. (2018). Guía práctica para el diseño y medición de competencias profesionales. ESIC Editorial.

Pérez, V. (2010). Universidad, ciudadanos y nómadas. Ediciones Nobel.

Pérez, N., Bisquerra, R., Filella, G.R., y Soldevila, A. (2010). Construcción del cuestionario de desarrollo emocional de adultos (QDE-A). Revista Española de Orientación y Psicopedagogía, 21(2), 367-379.

Pozo, J.A. (2017). Competencias profesionales: Herramientas de evaluación: el portafolios, la rúbrica y las pruebas situacionales. Narcea.

Pugh, G. y Lozano, A. (2019). El desarrollo de competencias genéricas en la educación técnica de nivel superior: un estudio de caso. Calidad en la Educación, 50, 143-179. https:// doi.org/10.31619/caledu.n50.725

Ramos, T. (Coord.) (2015). Universidades corporativas: diez casos de éxito. Editorial Universitat Oberta de Catalunya.
Ramos, T. (Coord.) (2017). Universidades corporativas: el reto digital. Editorial Universitat Oberta de Catalunya.

Ruiz, Y., García, M., Biencinto, C., y Carpintero, E. (2017). Evaluación de competencias genéricas en el ámbito universitario a través de entornos virtuales: Una revisión narrativa. RELIEVE, 32(2). http://doi. org/10.7203/relieve.23.1.7183

Sanjurjo, V.A. (2012). El Nuevo espacio Europeo de Educación Superior: Desafíos e Incertidumbres. Revista de Derecho UNED, 10, 585-609.

Spencer, L.M., \& Spencer, S.M. (1993). Competence at work: models for superior performance. John Wiley y Sons.

Villardón, L. (Coord.) (2015). Competencias genéricas en educación superior. Metodologías específicas para su desarrollo. Narcea.

UNESCO. (2015). Replantear la educación. ¿Hacia un bien común mundial? UNESCO. https://bit. ly/2CgJJnJf 


\section{Anexo 1. Guía docente de la asignatura sobre competencias genéricas}

\section{DATOS DE IDENTIFICACIÓN}

\begin{tabular}{|ll|l|}
\hline Curso académico: & 2019-2020 & \\
\hline Titulación: & Biotecnología & \\
\hline Rama de Conocimiento: & Ciencias & \\
\hline Facultad/Escuela: & Ciencias Experimentales & \\
\hline Asignatura: & Competencias Personales & \\
\hline Tipo: & Obligatoria & Créditos ECTS: \\
\hline Curso: & 1 & Código: \\
\hline Período docente: & Anual & \\
\hline Materia: & $\begin{array}{l}\text { Aspectos Sociales de la } \\
\text { Biotecnología }\end{array}$ & \\
\hline Módulo: & $\begin{array}{l}\text { Aspectos Sociales, Histó- } \\
\text { ricos y Económicos de la }\end{array}$ & \\
\hline Biotecnología & \\
\hline Idioma: & Presencial & \\
\hline $\begin{array}{l}\text { Total horas de dedica- } \\
\text { ción del alumno: }\end{array}$ & Castellano & \\
\hline Equipo Docente & 15 & Correo Electrónico \\
\hline & & \\
\hline
\end{tabular}

\section{DESCRIPCIÓN DE LA ASIGNATURA}

La asignatura pretende poner al alumno en camino hacia su madurez personal y profesional. Desde el descubrimiento de su vocación, provocar su respuesta a la misma dentro del ámbito universitario en el que se encuentra, potenciando el desarrollo de aquellas capacidades intelectuales, personales y sociales que faciliten su progreso y logren un profesional de la Biotecnología íntegro y capaz, preparado para liderar equipos de trabajo eficaces y comprometido socialmente. 
La asignatura pretende facilitar a los alumnos el desarrollo de principales competencias personales que configuran el perfil que la Facultad desea para sus alumnos. A través de las diferentes actividades individuales y de equipo que se llevan a cabo, cada alumno podrá valorar sus fortalezas y áreas de mejora para caminar hacia la excelencia, potenciando sus fortalezas y superando en comunidad sus debilidades. De este modo, el temario hace un recorrido por las distintas competencias personales críticas para el desempeño excelente de los alumnos en sus distintos ámbitos: personal, social, académico y profesional.

\section{OBJETIVO}

Profundizar en el conocimiento de sí mismo, reconociendo y forjando las competencias necesarias para fomentar su madurez personal y profesional.

Los fines específicos de la asignatura son:

- Que el alumno forme la mirada profunda y posibilitadora sobre la realidad.

- Que el alumno amplíe el conocimiento de sí mismo.

- Que el alumno descubra la importancia de trabajar en equipo.

- Que el alumno inicie la adquisición de la competencia de comunicación.

- Que el alumno descubra la importancia de comprometerse con su desarrollo personal.

\section{CONOCIMIENTOS PREVIOS}

Conocimientos complementarios con la asignatura de Filosofía Aplicada.

\section{CONTENIDOS}

Tema 1. Introducción.

1.1 Presentación de la asignatura: Aula, Mentoría y Proyecto Transversal.

1.2 Presentación de la Guía Docente.

Tema 2. Las competencias personales.

2.1 Historia de las competencias.

2.2 Definición del concepto de competencias personales.

2.3 Pilares de la competencia.

2.4 Las competencias personales en el contexto profesional.

2.5 Tipología de las competencias personales.

2.6 Proceso de la adquisición de competencias personales. 
Tema 3. Liderazgo de servicio como meta competencia.

3.1 Qué es el liderazgo y qué no lo es.

3.2 Funciones del líder.

3.3 Competencias del líder. Serán las competencias propias del temario.

3.4 Desarrollo del liderazgo.

Tema 4. Competencias intrapersonales.

4.1 Mirada profunda y proactividad (mentoría 1).

4.2 Conocimiento personal: la personalidad (mentoría 2), don (mentoría 4) y estilos de aprendizaje.

4.3 Superación personal: camino de desarrollo personal (mentoría 3), acciones CRECER y hábitos creativos.

4.4 Búsqueda de sentido: vocación y proyecto de vida (visión y misión) (mentoría 5 y 6 ).

Tema 5. Competencias cognitivas.

5.1 Pensamiento crítico y analítico.

5.2 Pensamiento creativo: la creatividad.

5.3 Toma de decisiones.

5.4 Gestión del tiempo y planificación.

Tema 6. Competencias interpersonales.

6.1 Trabajo en Equipo.

6.1.1 Diferencias entre equipo y grupo.

6.1.2 Dimensiones de un equipo.

6.1.3 Fases de desarrollo de un equipo.

6.1.4 Roles de Equipo.

6.2 Resolución de conflictos/negociación.

6.3 Comunicación.

6.3.1 Comunicación para el encuentro: presencia-escucha plena, empatía, asertividad y feedback.

6.3.2 Oratoria: presentaciones eficaces, lenguaje verbal, paraverbal y no verbal.

\section{ACTIVIDADES FORMATIVAS}

Nuestra metodología docente busca el desarrollo de las habilidades y competencias que formarán el modelo de persona y profesional que deseamos trasladar a la sociedad.

Para ello, trabajaremos:

1. En el aula, a través del trabajo individual y en equipo (proyecto transversal).

2. En las mentorías, a través de seis mentorías individuales de una hora de duración y los trabajos que se deriven de cada una de ellas. 


\section{DISTRIBUCIÓN DE LOS TIEMPOS DE TRABAJO}

\begin{tabular}{|c|c|}
\hline ACTIVIDAD PRESENCIAL & $\begin{array}{c}\text { TRABAJO AUTÓNOMO/ACTIVIDAD } \\
\text { NO PRESENCIAL }\end{array}$ \\
\hline 60 horas & 90 horas \\
\hline $\begin{array}{l}\text { - Clases magistrales. } 12 \mathrm{~h} \\
\text { - Trabajos (Trabajo en equipo). } 22 \mathrm{~h} \\
\text { - Aprendizaje personal tutorizado. } 6 \\
\text { Mentorías. 6h } \\
\text { - Exámenes. Evaluación-Presentacio- } \\
\text { nes. } 5 \mathrm{~h} \\
\text { - Seminarios, debates y talleres. } 15 \mathrm{~h}\end{array}$ & $\begin{array}{l}\text { - Trabajos y debates. Trabajo indivi- } \\
\text { dual. 20h } \\
\text { - Trabajos y debates. Trabajo en } \\
\text { equipo. } 40 \mathrm{~h} \\
\text { - Estudio teórico. } 20 \mathrm{~h} \\
\text { - Actividades complementarias de } \\
\text { formación científica, intelectual y } \\
\text { humana } 10 \mathrm{~h}\end{array}$ \\
\hline
\end{tabular}

\section{COMPETENCIAS}

\section{Competencias básicas}

Que los estudiantes hayan demostrado poseer y comprender conocimientos en un área de estudio que parte de la base de la educación secundaria general, y se suele encontrar a un nivel que, si bien se apoya en libros de texto avanzados, incluye también algunos aspectos que implican conocimientos procedentes de la vanguardia de su campo de estudio.

Que los estudiantes sepan aplicar sus conocimientos a su trabajo o vocación de una forma profesional y posean las competencias que suelen demostrarse por medio de la elaboración y defensa de argumentos y la resolución de problemas dentro de su área de estudio.

Que los estudiantes tengan la capacidad de reunir e interpretar datos relevantes (normalmente dentro de su área de estudio) para emitir juicios que incluyan una reflexión sobre temas relevantes de índole social, científica o ética.

Que los estudiantes puedan transmitir información, ideas, problemas y soluciones a un público, tanto especializado como no especializado.

Que los estudiantes hayan desarrollado aquellas habilidades de aprendizaje necesarias para emprender estudios posteriores con un alto grado de autonomía. 


\section{Competencias generales}

Habilidad para trabajar en equipo y gestionar grupos.

Capacidad para la resolución de problemas y la toma de decisiones.

Desarrollar la capacidad y el compromiso del propio aprendizaje y desarrollo personal. Desarrollar hábitos de comunicación oral y escrita.

\section{Competencias específicas}

Cultivar actitudes de liderazgo y responsabilidad social en el desempeño personal y profesional.

Identificar el propio proceso de maduración personal y las metas a alcanzar, organizando un plan de acción orientado a su consecución.

Capacidad de comunicar de forma oral y escrita los conocimientos adquiridos. Saber trabajar en equipo de modo efectivo y coordinado.

Desarrollar criterios para la resolución de problemas y la toma de decisiones tanto en el ámbito profesional como en el ámbito personal.

\section{RESULTADOS DE APRENDIZAJE}

Aprender a descubrirse a sí mismo en lo que hace y en cómo lo hace. Identificar algunas de sus fortalezas y debilidades.

Realizar un plan de desarrollo personal.

Conocer el proceso de comunicación y sus elementos.

Potenciar la creatividad en equipo.

Descubrir los roles dentro de un equipo.

Distinguir las diferentes formas de resolución de conflictos Descubrir las diferentes herramientas del pensamiento creativo.

Practicar la comunicación oral.

Distinguir los diferentes estilos de liderazgo.

Practicar una adecuada metodología de toma de decisiones. 


\section{SISTEMA DE EVALUACIÓN DEL APRENDIZAJE}

El sistema de evaluación, acorde con los objetivos de aprendizaje, busca principalmente incentivar el trabajo diario, la participación y el trabajo en equipo.

A.- Alumnos en primera matrícula

La nota final de la asignatura se compone de:

1. Pruebas de contenido y de adquisición de competencias: $40 \%$.

2. Proyecto transversal: $30 \%$.

3. Mentorías: $25 \%$.

4. Participación: $5 \%$.

Atención: La nota mínima en cada uno de los apartados que componen los distintos medios de evaluación, debe ser, al menos, de 4 puntos sobre 10.

B.- Alumnos con evaluación adaptada por razones justificadas, y alumnos en segundas y sucesivas convocatorias La nota final de la asignatura se compone de:

1. Prueba global de contenidos y de evaluación de adquisición de competencias: $45 \%$.

2. Presentación de proyecto transversal adaptado: $30 \%$.

3. Mentorías: $25 \%$.

Atención: La nota mínima en cada uno de los apartados que componen los distintos medios de evaluación, debe ser, al menos, de 4 puntos sobre 10 .

C.- Sistema de evaluación de alumno en convocatoria extraordinaria

1. Prueba global de contenidos y de evaluación de adquisición de competencias: $45 \%$.

2. Presentación de proyecto transversal: $30 \%$.

3. Presentación de portafolio de mentoría: $25 \%$.

Atención: La nota mínima en cada uno de los apartados que componen los distintos medios de evaluación, debe ser, al menos, de 4 puntos sobre 10 .

El Plagio en cualquiera de las entregas o trabajos que han de realizar los alumnos supondrá el suspenso del trabajo de que se trate y la apertura de un proceso disciplinario que puede implicar, entre otras consecuencias, el suspenso de la Asignatura. 


\section{BIBLIOGRAFÍA Y OTROS RECURSOS}

\section{Básica}

CARDONA, P. Creciendo como líder. EUNSA, Navarra, 2009.

COVEY, S. Los siete hábitos de la gente altamente efectiva. PAIDÓS, Barcelona, 2005. DOMÍNGUEZ PRIETO, X.M., Llamada y proyecto de vida, PPC, Madrid, 2007.

FRANKL, V. El hombre en busca del sentido, 1979.

MUÑOZ, C. CRESPÍ, P. ANGREHS, R. Habilidades sociales. 2011. Ediciones Paraninfo.

POLAINO, A. Aprender a escuchar, Planeta Testimonio, Barcelona, 2008.

\section{Complementaria}

EISENBERG, N. Y STRAYER, J. La Empatía y su desarrollo. Desclée de Brouwer, Bilbao, 1992.

RAMÓN-CORTÉS, F. La isla de los cinco faros. Un recorrido por las claves de la comunicación. RBA. Colección Nueva Empresa, Barcelona, 2010. 


\section{Anexo 2. Cuestionario sobre competencias genéricas}

Este cuestionario es anónimo. Tiene como objetivo conocer tu nivel de desarrollo en algunas competencias transversales importantes en la formación universitaria: conocimiento y desarrollo personal, trabajo en equipo y comunicación. Por favor, marca la opción con la que mejor te identifiques para cada item.

La escala de valoración es la siguiente:

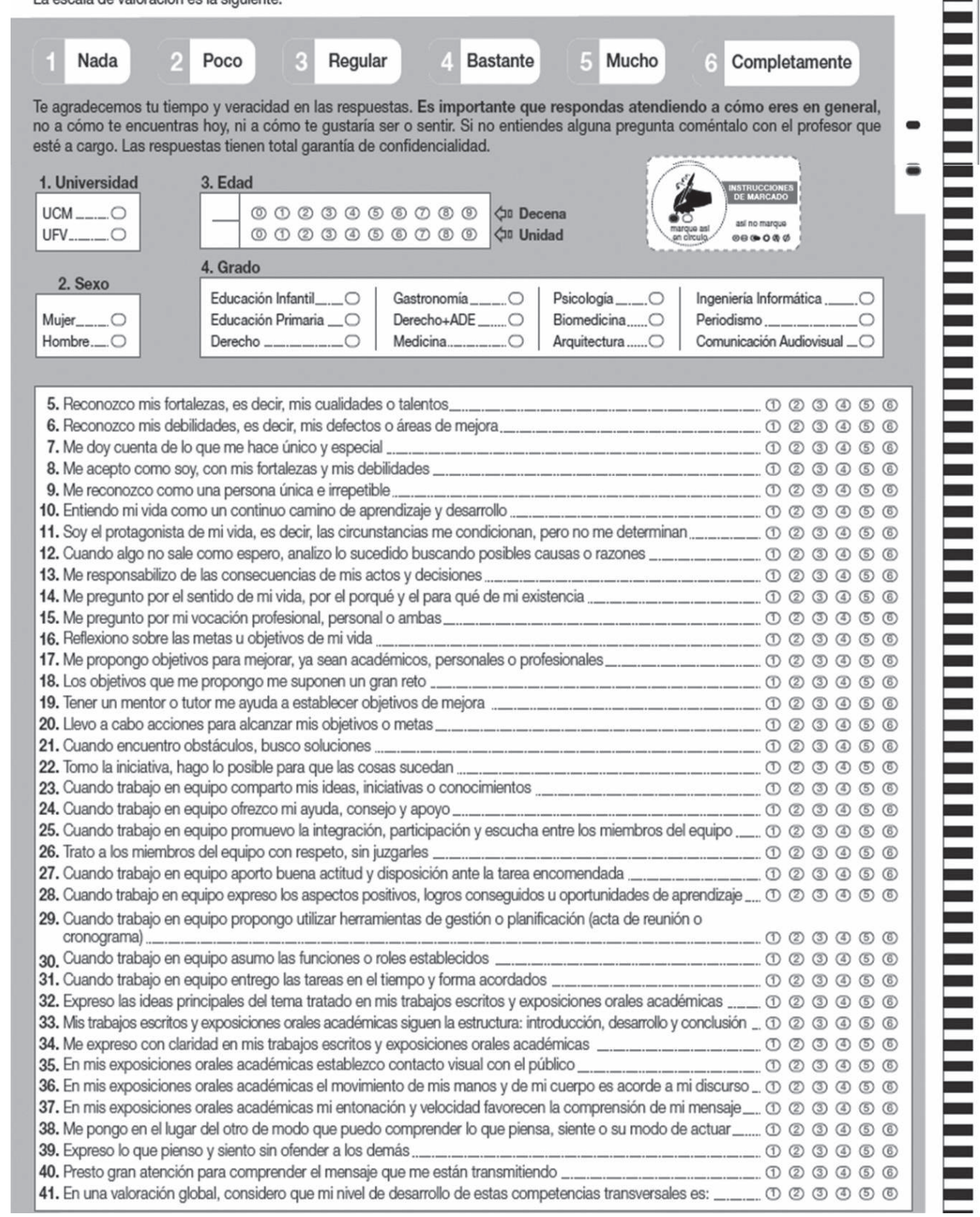




\section{PERFIL ACADÉMICO Y PROFESIONAL DE LOS AUTORES}

Paula Crespí. ORCID: http://orcid.org/0000-0002-3470-8424

Doctora en Educación por la Universidad Complutense de Madrid (UCM). Docente, Coach y Mentora en las áreas de Habilidades y Competencias Personales desde 2011. Responsable del Área de Formación e Investigación del Instituto de Acompañamiento de la UFV. Consultora de Recursos Humanos desde 2007. Sus principales líneas de Investigación son competencias, competencias transversales, acompañamiento y herramientas para el aprendizaje significativo. E-mail: p.crespi.prof@ufv.es

José Manuel García-Ramos. ORCID: http://orcid.org/0000-0002-56230900

Catedrático de Universidad. Doctor en Filosofía y C.C. de la Educación de la Universidad Complutense de Madrid. 4 sexenios de Investigación y 7 quinquenios de docencia reconocidos. Sus principales líneas de Investigación son Familia y Educación; Responsabilidad Social del Estudiante Universitario (RSEU) y Comunidades de aprendizaje. E-mail: jmgramos@ccedu.ucm.es

Fecha Recepción del Artículo: 17. Febrero. 2020

Fecha Modificación del Artículo: 04. Junio. 2020

Fecha Aceptación del Artículo: 08. Junio. 2020

Fecha Revisión para Publicación: 22. Junio. 2020 
